\title{
Properties of reaction fronts in a non-adiabatic two stage exothermic-endothermic competitive reaction scheme
}

\author{
W. Y. S. Wee ${ }^{1} \quad$ H. S. Sidhu ${ }^{2} \quad$ J. J. Sharples ${ }^{3}$ \\ I. N. Towers ${ }^{4}$ V. V. Gubernov ${ }^{5}$
}

(Received 29 October 2012; revised 5 September 2013)

\begin{abstract}
We numerically derive the properties of reaction fronts arising in a pre-mixed one dimensional two staged non-adiabatic competitive exothermic-endothermic reaction scheme where both reaction pathways compete for the same fuel. We utilise FlexPDE and the method of lines to obtain numerical solutions for properties such as the front speed and stability over a range of parameter values such as the Lewis number and the ratios of enthalpies and activation energies. Steady and pulsating speeds are demonstrated for specific regions of the parameter space.
\end{abstract}

http://journal.austms.org.au/ojs/index.php/ANZIAMJ/article/view/6302 gives this article, (C) Austral. Mathematical Soc. 2013. Published October 10, 2013, as part of the Proceedings of the 16th Biennial Computational Techniques and Applications Conference. ISSN 1446-8735. (Print two pages per sheet of paper.) Copies of this article must not be made otherwise available on the internet; instead link directly to this URL for this article. 
We also show that in some circumstances there exists a chaotic regime of combustion wave propagation.

Keywords: non-adiabatic; flame speed; combustion waves; competitive exothermic-endothemic

\section{Contents}

1 Introduction

C647

2 Mathematical model

C649

3 Numerical schemes

C652

4 Results

C654

5 Discussion

C659

References

C660

\section{Introduction}

Combustion processes have played a significant part in the advancement of technology and civilisation throughout human history. The multiple applications of combustion processes in today's industries, such as the synthesis of ceramics, combustion engines, and power generation, are the result of numerous studies and experiments [12].

Flames or combustion waves that propagate through a reactive media are of interest to industry, especially combustion waves that reflect travelling wave solutions. They find relevance in, for example, the synthesis of ceramics and advanced materials [17], power generation [19], and chemical reactors [2]. A combustion wave is essentially a propagating reaction front delineating the 
change from the initial chemical reactants to the reaction products. Whilst most observed combustion processes consist of multiple (perhaps tens or hundreds) chemical reactions, occurring consecutively or simultaneously, it is often useful to group reactions into simpler models describing the dominant reaction kinetics. Ignoring hydrodynamic effects, as is often done $[5,6,7,8$, $15,18,20,21]$, these reduced kinetic schemes give rise to reaction-diffusion systems that are more tractable to detailed mathematical analysis, and provide important insights into the overall process. For example, where thermal effects are prominent, the detailed kinetics can be reduced to a simple, useful model consisting of one exothermic and one endothermic reaction. Such systems are classified as: 'sequential', when both reactions occur consecutively [10, 13]; 'parallel', when both reactions occur at the same time whilst consuming different reactants [2]; and 'competitive', when both reactions are simultaneously occurring and feeding on the same reactant.

While sequential and parallel schemes are relatively well studied, there are few studies focused on kinetic schemes with competing endothermic and exothermic reactions, despite such schemes being relevant to decomposition and pyrolysis processes [1] and applications involving ammonium nitrate based explosives [16]. A recent study by Hmaidi et al. [8] investigated the existence of propagating combustion waves in a competitive, adiabatic, one dimensional reactive solid slab (infinite Lewis number), assuming high activation energies and an activation energy for the endothermic reaction twice that of the exothermic reaction. Sharples et al. [15] investigated differing regimes of wave propagation in a similar system assuming the same ratio of activation energies, but extending the investigation to non-infinite Lewis numbers, thus accommodating non-solid reactants within the analysis. Gubernov et al. [6] investigated the properties of combustion waves and travelling wave solutions in a similar adiabatic competitive system, applying asymptotic analysis and establishing the existence of endothermic, exothermic and mixed endothermic-exothermic parameter regimes. Wee et al. [21] considered a similar competitive adiabatic system with a more balanced contribution from the endothermic reaction for generic reactants, and established regimes of 
steady and pulsating propagating speeds. Certain behaviours, such as the loss of stability of the reaction front in combustion reaction schemes occur in certain parameter ranges, and result in possibly undesired outcomes in industrial applications [11].

The aim here is to investigate the behaviour and stability of the reaction fronts resulting from a competitive endothermic-exothermic scheme with generic reactants or fuel and to study the effects of heat loss to the ambient surroundings, thus extending recent research $[6,8,15,21]$ on adiabatic competitive endothermic-exothermic schemes to non-adiabatic settings. We utilise FlexPDE [4] to determine the properties of the propagating wave fronts, and verify the results obtained using the method of lines [14] implemented with Matlab.

\section{Mathematical model}

We consider a reaction scheme with two stage kinetics for combustion wave propagation in a pre-mixed one dimensional reactive medium. In contrast to previous studies $[6,8,15,21]$, we include the effects of volumetric heat loss due to Newtonian cooling.

The model is implemented in a way similar to that used by Turcotte et al. [18], where combustion is initiated via a hot wire ignition. As with previous studies $[6,8,15]$, we neglect hydrodynamic effects in the system. The model allows Lewis number variations so permits non-solid reactants, and we assume parameter values from previous studies $[15,21]$ to allow comparison between adiabatic and non-adiabatic systems. It is assumed that the reactant undergoes one of two possible competing reaction pathways: one endothermic and one exothermic; and that the reaction products are chemically inert and do not change the physical properties such as the heat capacities, density or diffusivity. An example of such a configuration is a long insulated cylinder containing a fuel undergoing decomposition, with an appropriate a priori 
averaging over the transverse spatial dimension of the reaction front. Such a set up was used in laboratory experiments studying the burning of ammonium nitrate $\left(\mathrm{NH}_{4} \mathrm{NO}_{3}\right)$ in the context of emulsion explosives [3, 18]. For instance, a decomposition reaction of ammonium nitrate modelled by two competing endothermic and exothermic reactions is

$$
\begin{aligned}
& \mathrm{NH}_{4} \mathrm{NO}_{3} \rightarrow \mathrm{NH}_{3}+\mathrm{HNO}_{3} \quad \text { (endothermic) } \\
& \mathrm{NH}_{4} \mathrm{NO}_{3} \rightarrow \mathrm{N}_{2} \mathrm{O}+2 \mathrm{H}_{2} \mathrm{O} \quad \text { (exothermic). }
\end{aligned}
$$

With the aim of analysing the properties of a combustion wave front in a competitive reaction-diffusion scheme in one spatial dimension, we propose a generalised description of the reaction pathways within such a scheme, assuming Arrhenius kinetics:

$$
\begin{array}{ll}
\text { Reactant } A \stackrel{k_{1}(T)}{\longrightarrow} \text { Product } A-\text { Heat }\left(Q_{1}\right), & k_{1}=A_{1} e^{-E_{1} / R T}, \\
\text { Reactant } A \stackrel{k_{2}(T)}{\longrightarrow} \text { Product } B-\operatorname{Heat}\left(Q_{2}\right), & k_{2}=A_{2} e^{-E_{2} / R T} .
\end{array}
$$

The exothermic reaction, indicated by the subscript 2 , drives the combustion wave front and is characterised by its activation energy $E_{2}\left(\mathrm{~J} \mathrm{~mol}^{-1}\right)$, preexponential rate constant $A_{2}\left(\mathrm{~s}^{-1}\right)$ and heat release $\mathrm{Q}_{2}\left(\mathrm{~J} \mathrm{~kg}^{-1}\right)$. Heat from the system is lost via the endothermic reaction, indicated by the subscript 1 , with activation energy $E_{1}\left(\mathrm{~J} \mathrm{~mol}^{-1}\right)$, pre-exponential rate constant $A_{1}\left(\mathrm{~s}^{-1}\right)$ and heat release $\mathrm{Q}_{1}\left(\mathrm{~J} \mathrm{~kg}^{-1}\right)$. The temperature and reaction rates of the endothermic and exothermic reactions are $\mathrm{T}(\mathrm{K}), \mathrm{k}_{1}\left(\mathrm{~s}^{-1}\right)$ and $\mathrm{k}_{2}\left(\mathrm{~s}^{-1}\right)$, respectively.

On applying heat and mass balance to the reaction and diffusion of reactant and heat, the governing equations for the competitive system are [20]

$$
\begin{aligned}
\rho c_{p} \frac{\partial T}{\partial t} & =k \frac{\partial^{2} T}{\partial x^{2}}+\rho\left(-Q_{1} A_{1} e^{-E_{1} / R T}+Q_{2} A_{2} e^{-E_{2} / R T}\right) C-\frac{h S}{V}\left(T-T_{a}\right), \\
\rho \frac{\partial C}{\partial t} & =\rho D \frac{\partial^{2} C}{\partial x^{2}}-\rho\left(A_{1} e^{-E_{1} / R T}+A_{2} e^{-E_{2} / R T}\right) C,
\end{aligned}
$$


where the reactant mass fraction and temperature are denoted by $\mathrm{C}$ and $\mathrm{T}$, respectively, with $T_{a}$ the ambient temperature. The time and space coordinates are $t$ and $x$, respectively. The density is $\rho\left(\mathrm{kg} \mathrm{m}^{-3}\right), \mathrm{k}\left(\mathrm{J} \mathrm{s}^{-1} \mathrm{~m}^{-1} \mathrm{~K}^{-1}\right)$ is the thermal conductivity, $\mathrm{D}\left(\mathrm{m}^{2} \mathrm{~s}^{-1}\right)$ is the coefficient of mass diffusion, $\mathrm{R}$ is the universal gas constant (equal to $\left.8.314 \mathrm{~J} \mathrm{~mol}^{-1} \mathrm{~K}^{-1}\right), \mathrm{c}_{\mathrm{p}}\left(\mathrm{J} \mathrm{kg}^{-1} \mathrm{~K}^{-1}\right)$ is the specific heat capacity at constant pressure of the reactant, $h\left(\mathrm{~J} \mathrm{~s}^{-1} \mathrm{~m}^{-2} \mathrm{~K}^{-1}\right)$ the Newton cooling coefficient and $S / \mathrm{V}$ the surface to volume ratio of the reacting vessel.

For convenience, we introduce the dimensionless temperature, space and time coordinates [15]

$$
u=\frac{R T}{E_{2}}, \quad x^{\prime}=x \sqrt{\frac{\rho Q_{2} A_{2} R}{k E_{2}}}, \quad t^{\prime}=t \frac{Q_{2} A_{2} R}{c_{p} E_{2}} .
$$

The dimensionless system of equations (with the primes omitted) is thus

$$
\begin{aligned}
\frac{\partial u}{\partial t} & =\frac{\partial^{2} u}{\partial x^{2}}+C e^{-1 / u}-q r C e^{-f / u}-l\left(u-u_{a}\right), \\
\frac{\partial C}{\partial t} & =\frac{1}{L e} \frac{\partial^{2} C}{\partial x^{2}}-\beta C e^{-1 / u}-\beta r C e^{-f / u}
\end{aligned}
$$

where $\mathfrak{u}_{\mathrm{a}}$ is the dimensionless ambient temperature, and the new parameters are

$$
q=\frac{Q_{1}}{Q_{2}}, \quad f=\frac{E_{1}}{E_{2}}, \quad r=\frac{A_{1}}{A_{2}}, \quad L e=\frac{k}{\rho c_{p} D}, \quad \beta=\frac{c_{p} E_{2}}{R Q_{2}}, \quad l=\frac{h S E_{2}}{\rho V R Q_{2} A_{2}} .
$$

Following the parameterisation of Sharples et al. [15], $\mathrm{q}$ is the ratio of the reaction enthalpies, $f$ is the ratio of activation energies, $r$ is the ratio of preexponential rate constants, Le is the Lewis number describing the ratio of the thermal conductivity and molecular diffusivity, $\beta$ is a parameter describing the energy balance of the exothermic reaction within the system (known as the exothermicity parameter), and $l$ is the non-dimensional heat loss coefficient incorporating a Newtonian heat loss term, $h$. The inclusion of $l$ allows the 
model to simulate non-adiabatic conditions, as compared to the adiabatic condition imposed elsewhere [6]. The Lewis number has two significant values: Le $\rightarrow \infty$ for solid fuels and Le $=1$ for gaseous fuels. For increasing values of $\beta$ the system becomes less exothermic.

The boundary conditions for the system, corresponding to a reaction front propagating in the positive $x$ direction, are

$$
\begin{aligned}
u & =u_{a}, \quad C=1, & \text { as } x \rightarrow+\infty ; \\
\frac{\partial u}{\partial x} & =0, \quad \frac{\partial C}{\partial x}=0, & \text { at } x=0 .
\end{aligned}
$$

On the right boundary $(x \rightarrow+\infty)$ is the cold $\left(u=u_{a}\right)$ and unburnt state $(\mathrm{C}=1)$ and $\mathrm{u}_{\mathrm{a}}=0$, as with previous studies $[6,7,15]$. On the left boundary $(x=0)$ is the burnt product mixture. However, neither the concentration nor the temperature of the product mixture can be specified explicitly. Since no reaction occurs on the left boundary, we set the derivatives of $u$ and $C$ to zero at $x=0$.

\section{Numerical schemes}

FlexPDE [4] is a finite element software package for obtaining numerical solutions to partial differential equations (PDEs). As a space and time adaptive finite element package, it minimises errors to a relative error tolerance level. We control the accuracy of the solutions by setting the value of error toleranceknown as the 'errlim' variable within the code. FlexPDE estimates the relative uncertainty among the solution variables of the PDE integrals over the mesh cell and compares it to the value of the 'errlim' variable. When this tolerance level is reached or exceeded, the mesh size is refined and the solution is re-iterated until the estimated errors in the variables are less than the set tolerance level. All solutions to the PDEs in this article were obtained using this package with the error tolerance limit set to errlim $=1 \times 10^{-5}$; that 


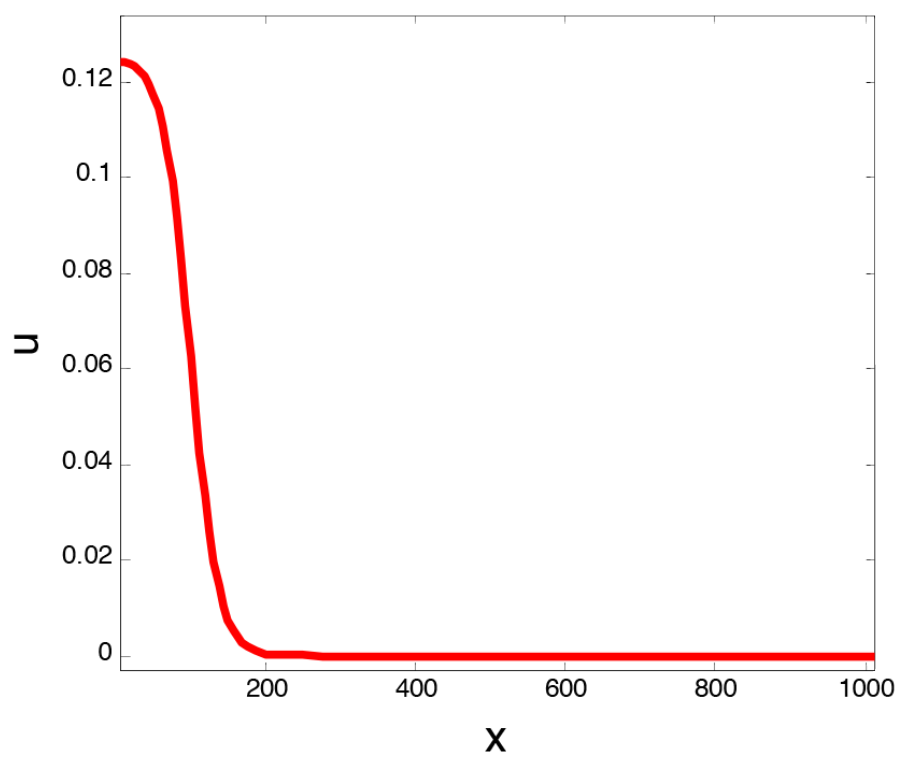

Figure 1: Initial conditions for the temperature profile for the domain $0 \leqslant$ $x \leqslant 1000$ showing the Gaussian pulse simulating a 'hot wire' ignition. The temperature profile remains flat for $x \geqslant 1000$.

is, FlexPDE refines the mesh cell until the estimated error in all variables (relative to the variable range) is less than $0.001 \%$ in each cell.

The method of lines (MOL) is a technique for obtaining numerical solutions to PDEs, and is well used in multiple studies [14]. In the MOL the spatial partial derivatives are discretised by finite difference approximations, transforming the PDEs into ordinary differential equations (ODEs), which are continuous in the time variable. The resulting system of ODEs is then solved as an initial value problem. In this analysis, the MOL is coded in Matlab and utilises the ode15s solver.

For the numerical integration of the governing PDEs, the initial condition for the reactant mass fraction was $C=1$, while the initial temperature 
was provided in the form of a Gaussian pulse simulating a hot wire ignition at $x=0$, for all numerical simulations. This is shown in Figure 1. The integration domain was set as $0 \leqslant x \leqslant 5 \times 10^{4}$ in order to accommodate the combustion wave profile, which may take some time to stabilize after some initial transient behaviour. Following previous work [6, 15], the dimensionless ambient temperature was set to zero [7] with the dimensionless volumetric heat loss coefficient set to $1 \times 10^{-6}$. The dynamics of the propagating wave are traced over a time interval of the order $10^{6}$. As with previous studies on competitive systems we always assume $f=1.5, q=r=1$, focusing on the behaviour of the system as Le and $\beta$ are varied.

\section{Results}

The governing equations (4) subject to the boundary conditions (6) were solved numerically using FlexPDE [4] and independently verified using MOL.

The typical fuel and temperature profile of the propagating reaction front over the domain of integration obtained from FlexPDE [4] is plotted in Figure 2. The reaction front occurs at approximately $x=2.5 \times 10^{4}$ and propagates from left to right. Ahead (to the right) of the front where the unreacted initial reactants are, the reactant mass fraction remains at $C=1$ and the temperature $\mathfrak{u}=\mathfrak{u}_{\mathrm{a}}$. Behind the reaction front where the reactants have been burnt, the reactant mass fraction decreases to $C=0$ and the temperature profile shows a temperature decay to the ambient temperature following the sharp increase at the reaction front.

Figure 3 shows discrete versions of the Hopf and extinction loci obtained from observations of the wave speed as determined by FlexPDE [4] for $1 \leqslant$ Le $\leqslant 10$. The green and blue curves demarcate the Hopf and extinction loci, respectively, while the red and black curves mark the locus for period doubling bifurcations. The Hopf locus is the set of points beyond which pulsations in the wave front occur, while the extinction locus is the set of points beyond which FlexPDE 


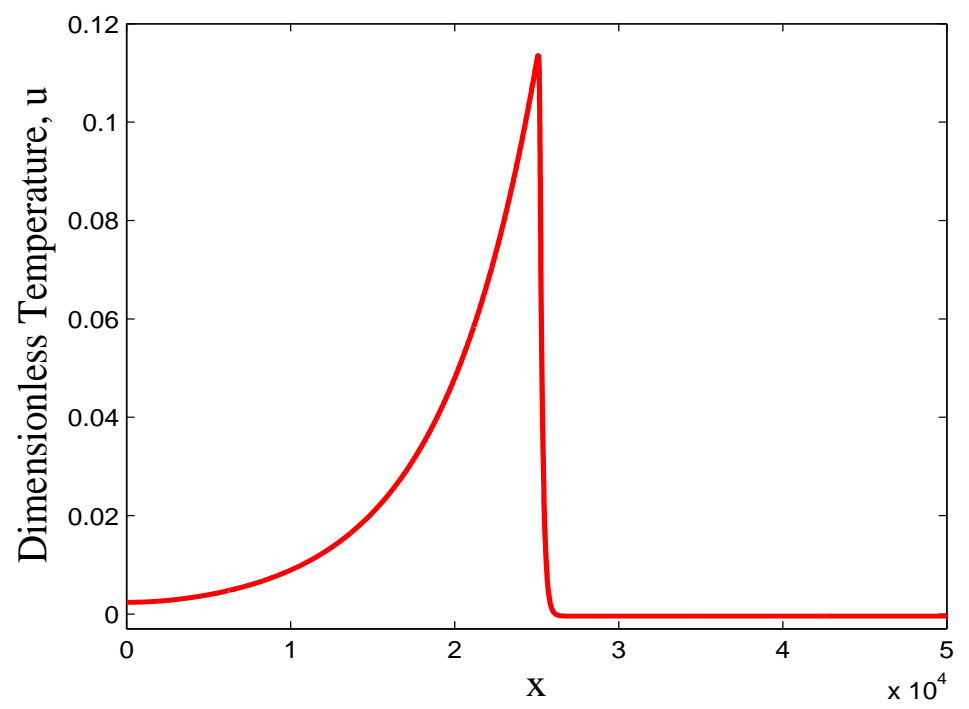

(a)

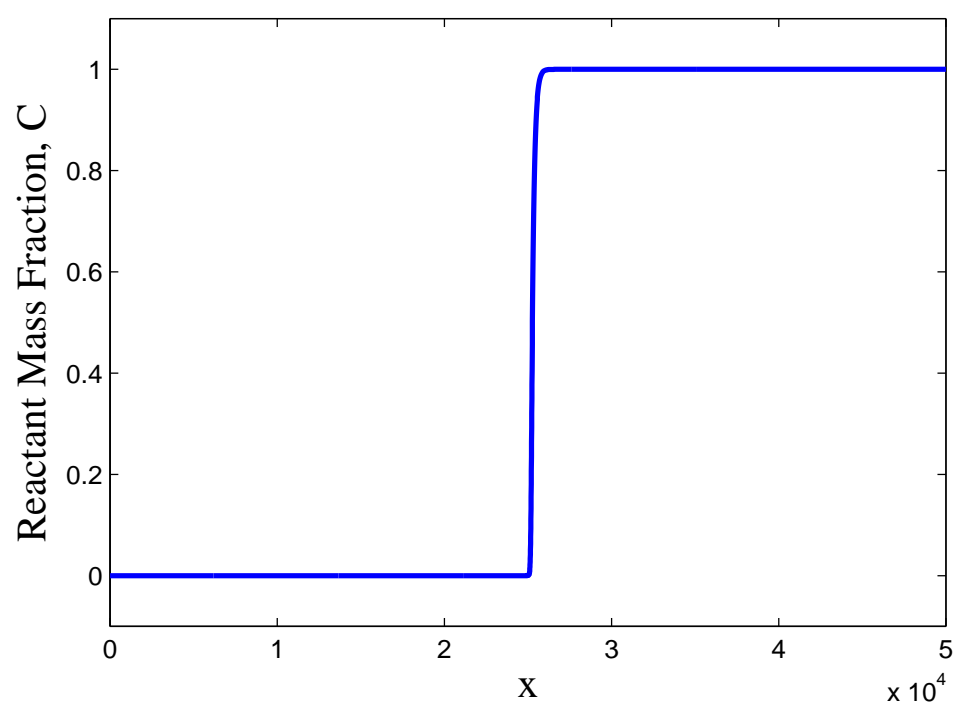

(b)

Figure 2: Profiles of the (a) temperature and (b) reactant mass fraction of the propagating combustion front for $\mathrm{Le}=2, \beta=7$ obtained using FlexpDE [4]. 


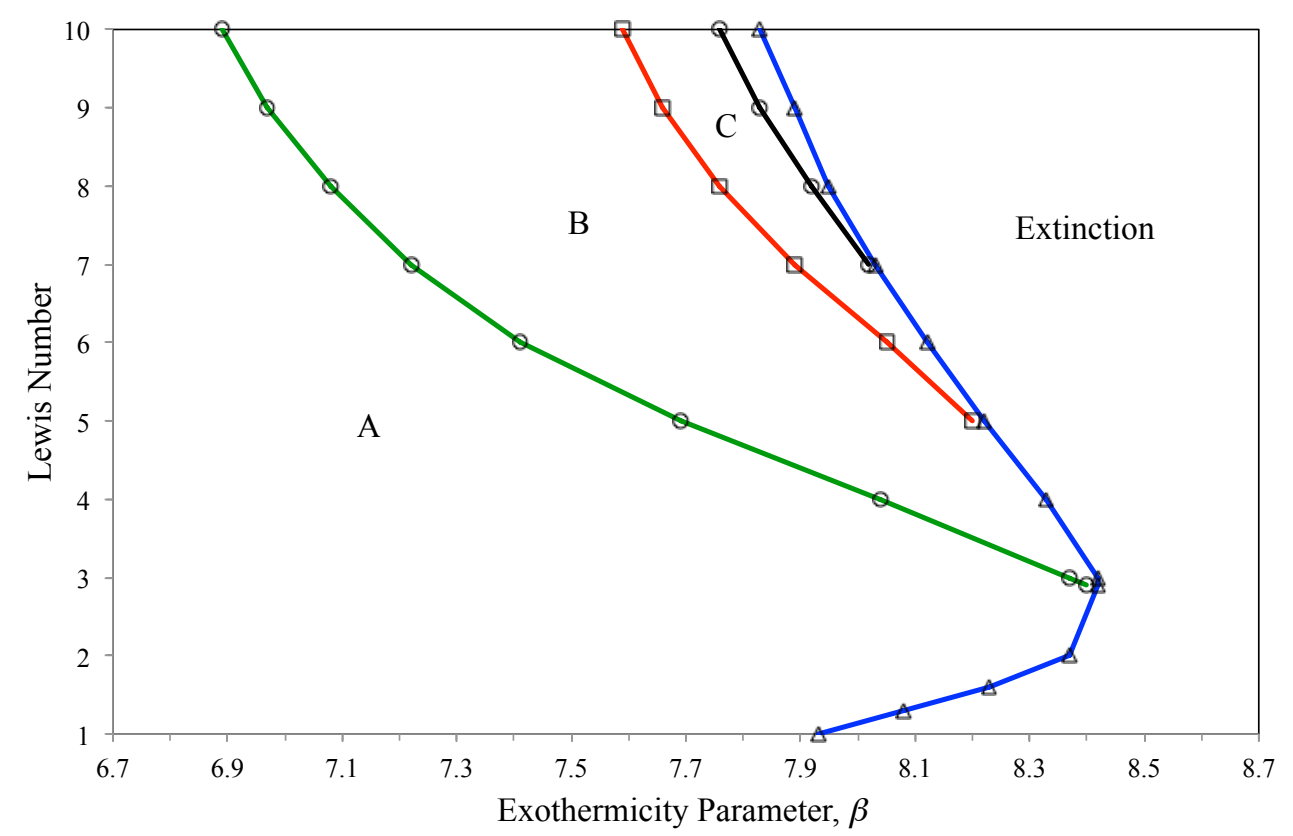

Figure 3: Bifurcation diagram showing the Hopf (green), extinction (blue) and period doubling bifurcation (red and black) loci for $1 \leqslant$ Le $\leqslant 10$. Steady propagating waves were found within region $\mathrm{A}$, period-one oscillations within region $\mathrm{B}$, period-two oscillations within region $\mathrm{C}$ and non-propagation beyond the extinction loci. The curves were obtained via FlexPDE [4] and the circular markers via the method of lines.

was not able to discern a non-trivial solution [15]. In our numerical analysis, we obtained a stable regime of propagating wave fronts with unique speeds in region $\mathrm{A}$, a steady pulsating regime of period-one and period-two oscillations in region $\mathrm{B}$ and $\mathrm{C}$, respectively, and extinction beyond the extinction loci. Period-four and chaotic oscillations were also detected in the region between region $\mathrm{C}$ (black curve) and the extinction region.

For small Lewis numbers, the behaviour of the wave front transitions directly from a steady speed regime to extinction as the value of $\beta$ is increased, 
observed in Figure 3 for Lewis numbers of approximately Le $\leqslant 3$. The Hopf locus intersects the extinction locus at Le $\approx 3$. However, as the magnitude of the Lewis number increases, a shift in the behaviour of the propagating wave fronts occurs and the fronts exhibit period-one, period-two and period-four pulsations. For instance, considering $\mathrm{Le}=8$, for a sufficiently small value of $\beta$ (e.g. $\beta=7)$, we obtain a propagating reaction front with a steady speed of $v \approx 0.021$, as seen in Figure 4a, corresponding to region A in Figure 3. As we increase the value of $\beta$ to beyond the Hopf bifurcation point of $\beta \approx 7.08$, the reaction front loses stability and manifests as pulsations with cyclical oscillations of the wave speed. Figure $4 \mathrm{~b}$ illustrates the oscillatory behaviour of the propagating wave for $\mathrm{Le}=8$ and $\beta=7.6$, corresponding to region $\mathrm{B}$ in Figure 3. This period-one type oscillation is characterised by a constant amplitude and period in the wave speed profile.

Further increasing $\beta$, we observe another bifurcation in the form of a period doubling from period-one to period-two pulsations, represented in Figure 4c, where the wave speed profile oscillates with a constant period between four wave speeds. Similar qualitative results were obtained in previous studies of adiabatic competitive endothermic-exothermic reactive schemes [15, 21] where both period-one and period-two pulsations were observed.

A further period doubling to period-four oscillations is observed as $\beta$ is increased, as shown in Figure $4 \mathrm{~d}$ for $\mathrm{Le}=8$ and $\beta=7.93$. In contrast, in previous work on the adiabatic competitive reaction scheme, only period-one and period-two oscillations were observed. Whilst no further period doubling bifurcations are detected in the non-adiabatic system studied here, complex oscillations, most likely chaotic, were found in the wave speed profile prior to the extinction of the reaction front. This is shown in Figure 4e. We speculate that a period doubling route to chaos, similar to that observed by Gubernov et al. [5], is occurring in a narrow region of parameter space, which will be the subject of future investigation. 


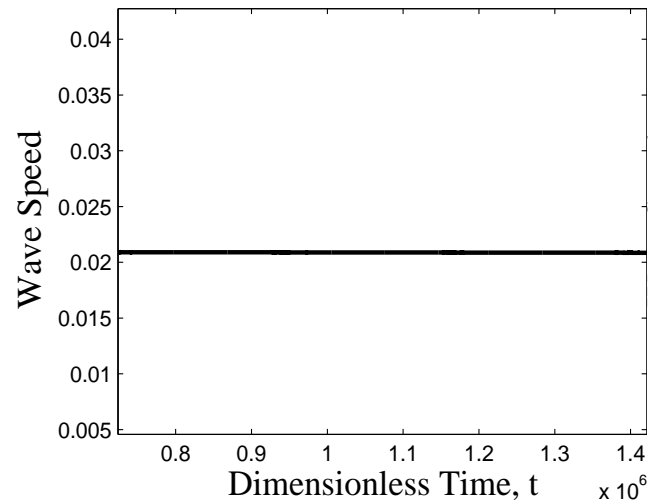

(a)

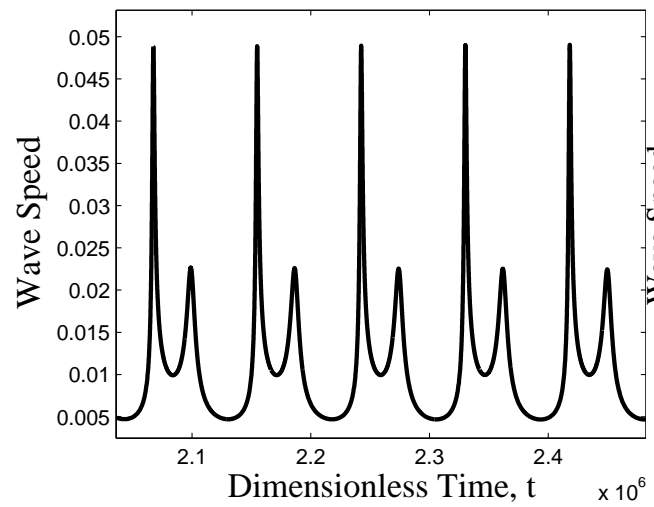

(c)

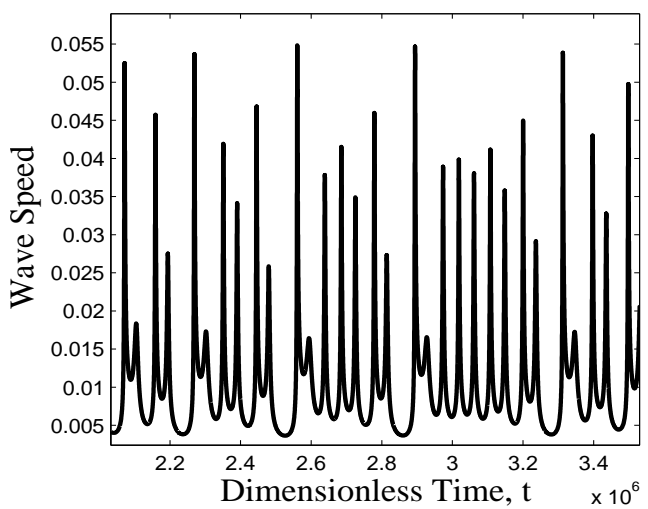

(e)

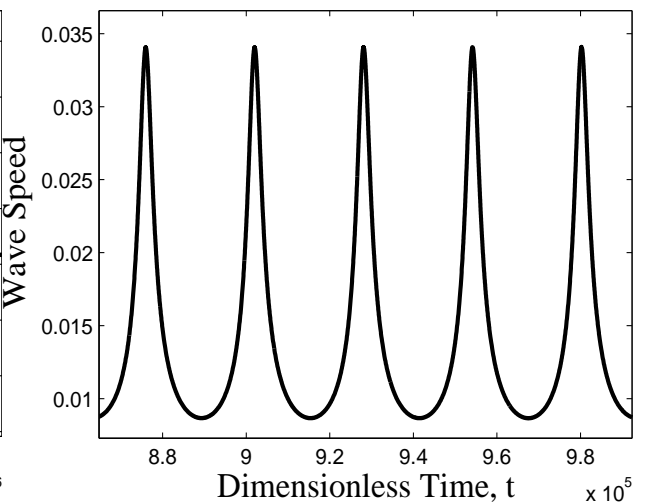

(b)

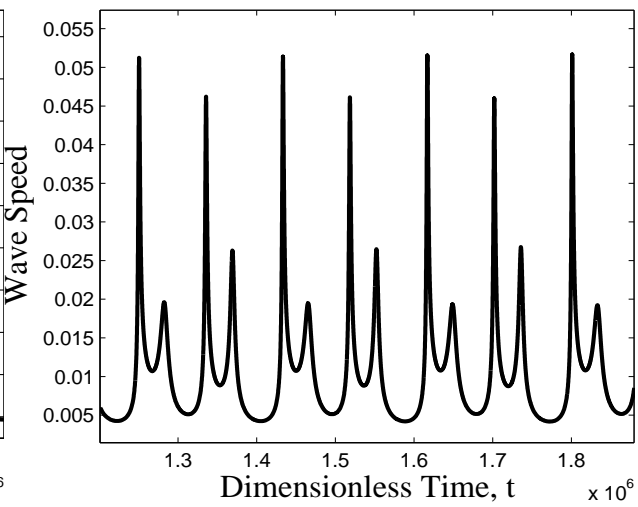

(d)

Figure 4: Wave speed profiles derived for $\mathrm{Le}=8$ and (a) $\beta=7$, (b) $\beta=7.6$, (c) $\beta=7.9$, (d) $\beta=$ 7.93 and (e) $\beta=7.95$. 


\section{Discussion}

We investigated the propagation of combustion waves in a competitive endothermic-exothermic reaction scheme under non-adiabatic conditions in one spatial dimension. The model was analysed numerically for $f=1.5$ and $r=q=1$ using the adaptive finite element package FlexPDE [4] for the numerical integration of the governing partial differential equations, and the results corroborated by an independent numerical scheme utilising the method of lines. Both methods yielded results which were qualitatively identical but with small quantitative differences in the wave speed profiles as well as in the Hopf bifurcation, period doubling bifurcations and extinction loci. Despite the simple chemistry assumed, complex behaviour was observed for specific parameter ranges. The results obtained show clear regions of steady speeds and differing modes of pulsating propagation. In particular, the existence of period-one, two and four pulsation regimes were established. For certain parameter regions, complex oscillations which were likely to be chaotic were also detected prior to the numerical extinction of the propagating wave front. Whilst period-one and two oscillations were previously detected in a similar competitive scheme under adiabatic conditions [15, 21], period-four and chaotic oscillations are yet to be detected in such competitive reaction schemes. The appearance of higher-order oscillations with the introduction of heat loss suggests that the presence of heat loss could be the cause of what appears to be a period doubling route to chaos and subsequent extinction that was noted in previous research [5].

The present study on a competitive endothermic-exothermic reaction scheme only considered chemical effects under the assumption of Arrhenius kinetics, together with Newtonian heat loss; other effects such as hydrodynamic interactions were ignored. However, the results obtained raise questions about the influence of oscillating reaction fronts arising in multi-stage reaction schemes on the dynamics associated with flame acceleration and transitions from deflagration to detonation [9]. Such questions are particularly pertinent given the relevance of the competitive endothermic-exothermic reaction scheme to 
ammonium nitrate based combustion. These questions will be considered in our ongoing research.

Acknowledgements This work was supported by the Australian Research Council grant 0878146.

\section{References}

[1] M. J. Antal and G. Varhegyi, Cellulose Pyrolysis Kinetics - The Current State of Knowledge, Industrial \& Engineering Chemistry Research, 34(3):703-717, 1995 doi:10.1021/ie00042a001 C648

[2] R. Ball, A. C. McIntosh and J. Brindley, Thermokinetic Models for Simultaneous Reactions: a Comparative Study, Combustion Theory and Modelling, 3(3):447-468, 1999. doi:10.1088/1364-7830/3/3/302 C647, C648

[3] S. K. Chan and R. Turcotte, Onset Temperatures in Hot Wire Ignition of AN-Based Emulsions, Propellants Explosives Pyrotechnics, 34(1):41-49, 2009. doi:10.1002/prep.200700288 C650

[4] FlexPDE ${ }^{\mathrm{TM}}, P D E$ Solutions Inc, http://www . pdesolutions . com. C649, C652, C654, C655, C656, C659

[5] V. V. Gubernov, A. Kobolov, A. Polezhaev and H. Sidhu, Period Doubling and Chaotic Transient in a Model of Chain-Branching Combustion Wave Propagation, Proceedings of the Royal Society A, 2011. doi:10.1098/rspa.2009.0668 C648, C657, C659

[6] V. V. Gubernov, J. J. Sharples, H. S. Sidhu, A. C. McIntosh and J. Brindley, Properties of Combustion Waves in the Model with Competitive Exo- and Endothermic Reactions, Journal of Mathematical 
Chemistry, 50(8):2130-2140, 2012. doi:10.1007/s10910-012-0021-y C648, C649, C652, C654

[7] V. V. Gubernov, H. S. Sidhu, G. N. Mercer, The Effect of Ambient Temperature on the Propagation of Nonadiabatic Combustion Waves, Journal of Mathematical of Mathematical Chemistry, 37(2):149-162, 2005. doi:10.1007/s10910-004-1447-7 C648, C652, C654

[8] A. Hmaidi, A. C. McIntosh and J. Brindley, A Mathematical Model of Hotspot Condensed Phase Ignition in the Presence of a Competitive Endothermic Reaction, Combustion Theory and Modelling, 14(6):893-920, 2010. doi:10.1080/13647830.2010.519050 C648, C649

[9] D. A. Kessler, V. N. Gamezo and E. S. Oran, Simulations of Flame Acceleration and Deflagration-to-Detonation Transitions in Methane-Air Systems, Combustion and Flame, 157(11):2063-2077, 2010. doi:10.1016/j.combustflame.2010.04.011 C659

[10] F. Liu, D. L. S. McElwain and C. P. Please, Simulation of Combustion Waves for Two-Stage Reactions, Proceedings of the 8th Biennial Computational Techniques and Applications Conference (CTAC97), pages 385-392, 1998. C648

[11] A. Makino, Fundamental Aspects of the Heterogeneous Flame in the Self-Propagating High-Temperature Synthesis (SHS) Process, Progress in Energy and Combustion Sciences, 27(1):1-74, 2001. doi:10.1016/S0360-1285(00)00004-6 C649

[12] A. G. Merzhanov and E. N. Rumanov, Physics of Reaction Waves, Reviews of Modern Physics, 71(4):1173-1211, 1999. doi:10.1103/RevModPhys.71.1173 C647

[13] C. P. Please, F. Liu and D. L. S. McElwain, Condensed Phase Combustion Travelling Waves with Sequential Exothermic or Endothermic Reactions, Combustion Theory and Modelling, 7(1):129-143, 2003. doi:10.1088/1364-7830/7/1/307 C648 
[14] W. E. Schiesser, The numerical method of lines: Integration of Partial Differential Equations, Academic Press, Inc, 1991. C649, C653

[15] J. J. Sharples, H. S. Sidhu, A. C. Mcintosh, J. Brindley and V. V. Gubernov, Analysis of Combustion Waves Arising in the Presence of a Competitive Endothermic Reaction, IMA Journal of Applied Mathematics, 77(1):18-31, 2012. doi:10.1093/imamat/hxr072 C648, C649, C651, C652, C654, C656, C657, C659

[16] V. P. Sinditskii, V. Y. Egorshev, A. I. Levshenkov and V. V. Serushkin, Ammonium nitrate: Combustion Mechanism and the Role of Additives, Propellants Explosives Pyrotechnics, 30(4):269-280, 2005. doi:10.1002/prep.200500017 C648

[17] J. Subrahmanyam and M. Vijayakumar, Self-Propagating High-Temperature Synthesis, Journal of Materials Science, 27(23):6249-6273, 1992. doi:10.1007/BF00576271 C647

[18] R. Turcotte, S. Goldthorp, C. M. Badeen and S. K. Chan, Hot-Wire Ignition of AN-Based Emulsions, Propellants Explosives Pyrotechnics, 33(6):472-481, 2008. doi:10.1002/prep.200700276 C648, C649, C650

[19] S. Walia, R. O. Weber, K. Latham, P. Petersen, J. T. Abrahamson, M. S. Strano, and K. Kalantar-zadeh, Oscillatory Thermopower Waves Based on $\mathrm{Bi}_{2} \mathrm{Te}_{3}$ Films, Advanced Functional Materials, 21(11):2072-2079, 2011. doi:10.1002/adfm.201001979 C647

[20] R. O. Weber, G. N. Mercer, H. S. Sidhu and B. F. Gray, Combustion Waves for Gases $(\mathrm{Le}=1)$ and Solids $(\mathrm{Le} \rightarrow \infty)$, Proceedings of the Royal Society of London A, 453(1960):1105-1118, 1997. doi:10.1098/rspa.1997.0062 C648, C650

[21] W. Y. S. Wee, J. J. Sharples, H. S. Sidhu and V. V. Gubernov, Analysis of a Two-Stage Competitive Endothermic-Exothermic Reaction Scheme, Proceedings of the 40th Australian Chemical Engineering Conference (CHEMECA 2012), submitted June 2012. C648, C649, C657, C659 


\section{Author addresses}

1. W. Y. S. Wee, Applied and Industrial Mathematics Research Group, UNSW Canberra, the Australian Defence Force Academy, PO Box 7916, Canberra BC 2610, Australia.

2. H. S. Sidhu, Applied and Industrial Mathematics Research Group, UNSW Canberra, the Australian Defence Force Academy, PO Box 7916, Canberra BC 2610, Australia.

mailto:h.sidhu@adfa.edu.au

3. J. J. Sharples, Applied and Industrial Mathematics Research Group, UNSW Canberra, the Australian Defence Force Academy, PO Box 7916, Canberra BC 2610, Australia.

4. I. N. Towers, Applied and Industrial Mathematics Research Group, UNSW Canberra, the Australian Defence Force Academy, PO Box 7916, Canberra BC 2610, Australia.

5. V. V. Gubernov, I.E. Tamm Theory Department, P. N. Lebedev Physical Institute of Russian Academy of Sciences, 53 Leninskii Prospect, Moscow 119991, Russian Federation. 